\title{
Presentación clínica del golpe de calor en niños menores de 2 años en Lima - Perú.
}

\author{
LOZA MUNARRIZ Reyner, MIYAHIRA ARAKAKI Juan**, CIEZA ZEVALLOS Javier**.
}

\section{SUMMARY}

Objective: To describe the clinic manifestations of heat stroke in infants during EI Niño phenomena. Material and methods: We included 13 infants with heat stroke (HS) criteria, attended at the Hospital Nacional Cayetano Heredia between Jan $1^{\circ}$ and Mar 31, 1998. We registered hematocrito, coagulation tests, hepatic and renal function tests, blood gas analysis, serum and urinary electrolytes and urinary flux, intravenous solution used in hydrated therapy and outcome condition. Results: 4/13 died, 4/13 lived with serious neurological sequels. The clinic manifestations more important were convulsions $(\mathbf{7 7 \%})$, respiratory distress $(85 \%)$, water deficit $(\mathbf{7 7 \%})$, shock $(61 \%)$, acute diarrhea $(85 \%)$ and polyuria $(75 \%)$. In the laboratory test, was found anemia $(84 \%)$, leukocytosis $(60 \%)$, prolonged prothrombin time $(53 \%)$, hyperglycemia $(53 \%)$, elevated serum aminotransferases $(83 \%)$, hyponatremia $(84 \%)$, hypokalemia $(70 \%)$, elevated serum creatinine $(\mathbf{8 4} \%)$, elevated serum urea $(53 \%)$ and metabolic acidosis $(\mathbf{1 0 0 \%})$. Statistic association was found only among prolonged prothrombin time and use of hypertonic solutions in initial therapy with bad outcome condition. Conclusion: HS had grave prognostic which prolonged prothrombin time and use of hypertonic solutions in initial therapy were associated with died risk or grave neurologic sequels. ( Rev Med Hered 2001; 12: 113-119).

KEY WORDS: Heat stroke, infants, hyperthermia, hyponatremia, hypokalemia, metabolic acidosis, prothrombin time, polyuria, renal failure.

\section{RESUMEN}

Objetivo: Describir el cuadro clínico de lactantes con golpe de calor (GC) durante El Fenómeno del el Niño de 1998. Material y métodos: Se incluyeron 13 lactantes con criterios de GC, que acudieron a Emergencia Pediátrica del Hospital Cayetano Heredia entre el $1^{\circ}$ enero y el 31 marzo 1998. Se registró hematocrito, pruebas de coagulación, función hepática y renal, gasometría, electrolitos séricos y urinarios. Asimismo el flujo urinario, tipo de solución endovenosa utilizada para la rehidratación y la condición final del paciente al alta. Resultados: De los 13 lactantes, 4 fallecieron y 4 quedaron con secuelas neurológicas graves al alta. Los hallazgos clínicos relevantes fueron convulsiones $(77 \%)$, dificultad respiratoria (85\%), deshidratación (77\%), shock $(61 \%)$, diarrea aguda $(85 \%)$ y poliuria $(75 \%)$. En el laboratorio general: anemia (84\%), leucocitosis $(60 \%)$ tiempo de protrombina prolongado (53\%), hiperglicemia $(53 \%)$ elevación de transaminasas $(83 \%)$. En las alteraciones del medio interno; hiponatremia (84\%), hipokalemia 
(70\%), creatinina elevada (84\%), urea elevada (53\%) y acidosis metabólica (100\%). En el análisis sólo hubo asociación estadística entre el tiempo de protombina prolongado y el manejo inicial de fluidos con la condición final desfavorable. Conclusión: El GC es una enfermedad de pronóstico grave, donde el tiempo de protrombina prolongado, y el uso de soluciones hipertónicas en el manejo inicial, se asociaron a riesgo de muerte o secuelas neurológicas graves. Consideramos importante dar a conocer el comportamiento clínico y los factores asociados con un final catastrófico. ( Rev Med Hered 2001; 12: 113-119).

PALABRAS CLAVE: Golpe de calor, niños, hiponatremia, hipokalemia, acidosis metabólica, tiempo de protrombina, poliuria, insuficiencia renal.

\section{INTRODUCCION}

El golpe de calor es una entidad poco frecuente, fue descrita por primera vez en 1929 por Wakefield, quien recopiló datos históricos ocurridos en Pekin en 1743, donde fallecieron 11000 personas por efecto del intenso calor en el medio (1). En 1923, Adolph fue el primero en reconocer la patogénesis del golpe de calor (2); sin embargo, fue en 1953 cuando el Medical Research Council de Gran Bretaña estableció la clasificación internacional de la enfermedad por calor (3).

De esta forma, la enfermedad por calor quedó definida en tres condiciones de importancia dominante. $\mathrm{La}$ primera fue denominada calambres por calor, que son calambres musculares intensos en personas que ingieren agua sin sales y que se encuentran expuestos a ambientes con muy alta temperatura. La segunda, fue llamada agotamiento por calor, que es de dos tipos: una asociada a perdida de agua y la segunda asociada predominantemente a depleción de sal; ocurre durante ondas de calor, cuando la persona realiza intenso trabajo muscular y se asocia a sudoración excesiva. La tercera forma, es el Golpe de calor, que es un desorden catastrófico caracterizado por hiperpirexia (temperatura rectal mayor de $41^{\circ} \mathrm{C}$ ), delirio, coma y falta de sudoración. Esta última presentación, es la menos común y la más importante. Ocurre en forma epidémica, se acompaña de alta mortalidad (80\%) y los sobrevivientes pueden quedar con secuelas neurológicas en forma definitiva (4).

Durante el año 1997, se detectó el calentamiento de las aguas del Océano Pacífico, como consecuencia del fenómeno de "El Niño". En la costa norte del Perú este fenómeno ocasionó grandes desastres naturales e inusual aumento de la temperatura del ambiente, en forma permanente para la región. Muchas viviendas fueron arrasadas por el desborde de los ríos y sus habitantes quedaron expuestos al sol en forma continua. En el mes de Febrero de 1998, la temperatura promedio en Lima fue de $31^{\circ} \mathrm{C}$, con picos de temperatura percibida por las personas de $35^{\circ} \mathrm{C}$ al medio día. El Hospital Nacional Cayetano Heredia, que se encuentra en Lima-Perú, atiende una población socioeconómicamente desfavorecida y en Febrero de 1998, atendió niños que acudieron con cuadros febriles durante los días de mayor temperatura y que se acompañaron de un cuadro clínico sugerente de Golpe de Calor.

Las descripciones hechas en la literatura médica sobre el golpe de calor en niños, no permiten establecer con claridad las características del cuadro clínico y las alteraciones del medio interno. Esto dificulta notablemente un adecuado diagnóstico y manejo de estos pacientes y puede gravitar en la elevada mortalidad observada. Por lo tanto creemos necesario conocer las alteraciones del medio interno, establecer guías clínicas y difundirlas en la comunidad médica, sobre todo en los lugares donde situaciones climáticas semejantes a lo que ocurrió en el Perú, pueden ser observadas. El objetivo del presente estudio fue describir las alteraciones del medio interno en los niños con diagnóstico de Golpe de Calor.

\section{MATERIAL Y METODOS}

En este estudio se incluyeron los pacientes lactantes que acudieron al servicio de Emergencia de Pediatría del Hospital Nacional Cayetano Heredia entre el $1^{\circ}$ de enero y el 31 de marzo de 1998 y que presentaron al ingreso hipertermia (temperatura rectal $>39^{\circ} \mathrm{C}$ en forma persistente por mas de 6 horas y que no cedían con medios físicos y/o antipiréticos), asociado a compromiso de conciencia, y/o presencia de convulsiones, deshidratación, shock, deposiciones diarreicas, distres respiratorio, falta de sudoración y/o sangrado. En los pacientes incluidos, se registró el estado de salud 15 días antes del ingreso al hospital y el tratamiento recibido (vía y tipo de líquidos, antipiréticos y/o antibióticos). El hematocrito, hemograma, tiempo de protrombina (TP), tiempo de tromboplastina parcial (TTP), glicemia, urea, creatinina, electrolitros séricos y urinarios, osmolalidad sérica y urinaria, magnesio sérico, proteínas totales y fraccionadas, gases sanguíneos y examen de orina completo, fueron evaluados al momento del ingreso. Además se registraron: peso y flujo de orina en las 
Tabla $\mathbf{N}^{\circ} 1$. Datos generales de los pacientes.

\begin{tabular}{lccccc} 
PACIENTE & $\begin{array}{c}\text { EDAD } \\
\text { (Meses) }\end{array}$ & $\begin{array}{c}\text { T. DE ENF. } \\
\text { (Horas) }\end{array}$ & SEXO & $\begin{array}{c}\text { Temp amb } \\
\text { sentida }\left({ }^{\circ} \mathrm{C}\right)\end{array}$ & $\begin{array}{c}\text { Estado clínico } \\
\text { previo }\end{array}$ \\
\hline FV & 8 & 144 & $\mathrm{~F}$ & 34.0 & Sana \\
VM & 10 & 72 & $\mathrm{M}$ & 34.0 & Retardo de desarrollo \\
$\mathrm{HC}$ & 10 & 168 & $\mathrm{~F}$ & 32.0 & Sana \\
$\mathrm{HM}$ & 5 & 48 & $\mathrm{~F}$ & 31.5 & Sana \\
TR & 6 & 60 & $\mathrm{M}$ & 26.5 & Sano \\
PR & 12 & 72 & $\mathrm{M}$ & 30.5 & Sano \\
MCH & 13 & 17 & $\mathrm{~F}$ & 30.5 & Sana \\
CCQ & 16 & 72 & $\mathrm{~F}$ & 35.2 & Sana \\
FC & 9 & 48 & $\mathrm{M}$ & 32.5 & Sano \\
LE & 8 & 168 & $\mathrm{M}$ & 32.7 & Sano \\
VM & 12 & 72 & $\mathrm{M}$ & 30.4 & Sano \\
BJ & 10 & 60 & $\mathrm{~F}$ & 32.6 & Sana \\
CHC & 7 & 72 & M & 32.7 & Sano \\
\hline
\end{tabular}

primeras 8 horas.

Se consideró la condición final del paciente al alta, definiéndose las categorías de vivo sin secuelas, vivo con secuelas neurológicas y fallecido. Con los datos de laboratorio registrados, se calculó el anion gap $(\mathrm{AG})(5,6)$. El tipo de disturbio ácido-base se definió de acuerdo a lo establecido universalmente (7).

El análisis de los datos se realizó usando estadística descriptiva y estadística analítica con el software EPI INFO v 6 y SPSS.

\section{RESULTADOS}

Se incluyeron a 13 lactantes entre 5 y 16 meses de edad quienes cumplían con los criterios de inclusión. En la tabla $\mathrm{N}^{\circ} 1$, se presentan los datos generales de los pacientes. Los hallazgos clínicos y del examen de orina más relevantes que presentaron los pacientes al momento de la admisión al hospital se pueden apreciar en la tabla $\mathrm{N}^{\circ} 2$. Los pacientes presentaron poliuria en el $75 \%$, dificultad respiratoria en el $85 \%$, diarrea $85 \%$, convulsiones $77 \%$, shock $62 \%$, sangrado $62 \%$ y anhidrosis en el $15 \%$.

Los datos de laboratorio hematológicos y bioquímicos más importantes, son presentados en la tabla $\mathrm{N}^{\circ} 3$. Los datos relacionados al medio interno se observan en las tablas $\mathrm{N}^{\circ} 4$ y $\mathrm{N}^{\circ} 5$. Las características de las soluciones utilizadas en el tratamiento inicial de los paciente, los días de permanencia hospitalaria y la condición final de los pacientes se muestran en la tabla $\mathrm{N}^{\circ} 6$.

En el análisis bivariado se encontró asociación entre el tiempo de protrombina (TP) y el manejo inicial de fluidos con una condición final desfavorable (Tabla $\mathrm{N}^{\circ} 7$ ).

\section{DISCUSION}

Los cambios climatológicos ocurridos en diversos lugares del planeta, han producido cambios notables en extensas regiones geográficas, que a su vez han originado situaciones mórbidas desconocidas hasta entonces en estas áreas.

En la costa peruana, el intenso calor de los primeros meses de 1998 por efecto del fenómeno de "El Niño", produjo un inesperado incremento de situaciones de gravedad diversa, relacionados a infecciones o simplemente a la persistencia de fiebre de cualquier etiología en grupos extremos de la vida (ancianos y lactantes) (8).

En el Hospital Nacional Cayetano Heredia, sólo se había descrito, la aparición de cuadros graves relacionados a síndromes febriles de alta temperatura en adultos, no así en niños y menos en lactantes.

En nuestro grupo de pacientes, no pudimos evidenciar enfermedad infecto-contagiosa, bacteriana 
Tabla N². Datos clínicos de los pacientes a la admisión al hospital.

\begin{tabular}{|c|c|c|c|}
\hline PCTE & $\begin{array}{l}\mathrm{T}^{\circ} \mathrm{RECTAL} \\
\quad\left({ }^{\circ} \mathrm{C}\right)\end{array}$ & $\begin{array}{l}\text { Flujo urinario (cc/Kg/h) y } \\
\text { Examen de orina }\end{array}$ & SIGNOS Y SINTOMAS EXISTENTES \\
\hline FV & 39.3 & $\begin{array}{l}\text { Qu } 6.2 \\
\text { Hemoglobinuria, albuminuria, } \\
\text { glucosuria }\end{array}$ & $\begin{array}{l}\text { Compromiso sensorio, convulsión dificultad respiratoria, EDA*, } \\
\text { sangrado }\end{array}$ \\
\hline VM & 39.5 & $\begin{array}{l}\text { Qu } 3.4 \\
\text { Albuminuria }\end{array}$ & Compromiso sensorio, convulsión, dificultad respiratoria, EDA *. \\
\hline $\mathrm{HC}$ & 39.0 & $\begin{array}{l}\text { Qu } 2.0 \\
\text { Hemoglobinuria } \\
\text { Albuminuria, glucosuria }\end{array}$ & Compromiso sensorio, deshidratación, convulsión, shock, EDA *. \\
\hline HM & 42.5 & Qu 3.7 & $\begin{array}{l}\text { Compromiso sensorio, deshidratación, convulsión, dificultad } \\
\text { respiratoria, shock, } E D A^{*} \text {, sangrado }\end{array}$ \\
\hline TR & 39.5 & $\begin{array}{l}\text { Qu } 10 \\
\text { Sin alteraciones }\end{array}$ & $\begin{array}{l}\text { Compromiso sensorio, deshidratación, convulsión, dificultad } \\
\text { respiratoria, shock, EDA*. }\end{array}$ \\
\hline PR & 41.0 & $\begin{array}{l}\text { Qu } 5.6 \\
\text { Albuminuria }\end{array}$ & $\begin{array}{l}\text { Compromiso sensorio, convulsión, dificultad respiratoria, shock, } \\
\text { EDA }^{*} \text {. }\end{array}$ \\
\hline $\mathrm{MCH}$ & 41.0 & $\begin{array}{l}\text { Qu } 5.8 \\
\text { Glucosuria }\end{array}$ & $\begin{array}{l}\text { Compromiso sensorio, deshidratación, convulsión, dificultad } \\
\text { respiratoria, anhidrosis, shock, EDA*. }\end{array}$ \\
\hline CCQ & 41.0 & $\begin{array}{l}\text { Qu } 2.2 \\
\text { Hemoglobinuria, Albuminuria, } \\
\text { glucosuria }\end{array}$ & $\begin{array}{l}\text { Compromiso sensorio, deshidratación, convulsión, dificultad } \\
\text { respiratoria, anhidrosis, EDA*. }\end{array}$ \\
\hline FC & 40.0 & $\begin{array}{l}\text { Qu... } \\
\text { Sin alteraciones }\end{array}$ & Compromiso sensorio, deshidratación, dificultad respiratoria, EDA *. \\
\hline LE & 40.8 & $\begin{array}{l}\text { Qu } 5.6 \\
\text { Hemoglobinuria }\end{array}$ & $\begin{array}{l}\text { Compromiso sensorio, deshidratación,convulsión, dificultad } \\
\text { respiratoria. }\end{array}$ \\
\hline VM & 40.0 & $\begin{array}{l}\text { Qu } 14 \\
\text { Hemoglobinuria } \\
\text { Albuminuria }\end{array}$ & Compromiso sensorio, deshidratación, anhidrosis, shock, EDA*. \\
\hline BJ & 39.5 & $\begin{array}{l}\text { Qu } 6.8 \\
\text { Hemoglobinuria, albuminuria, } \\
\text { glucosuria }\end{array}$ & $\begin{array}{l}\text { Compromiso sensorio, deshidratación, convulsión, dificultad } \\
\text { respiratoria, anhidrosis, shock, EDA* } \text {. }\end{array}$ \\
\hline $\mathrm{CHC}$ & 41.0 & $\begin{array}{l}\text { Qu } 1.2 \\
\text { Hemoglobinuria }\end{array}$ & Compromiso sensorio, deshidratación, dificultad respiratoria. \\
\hline
\end{tabular}


Tabla $N^{\circ} 3$. Principales resultados de laboratorio.

\begin{tabular}{|c|c|c|c|c|c|c|c|c|}
\hline PAC & $\begin{array}{c}\text { Hcto } \\
(\%)\end{array}$ & $\begin{array}{l}\text { Recuento } \\
\text { leucocit }\end{array}$ & $\mathrm{TP}$ & TTP & $\begin{array}{c}\text { Glicemia } \\
(\mathrm{mm} / \mathrm{Lt})\end{array}$ & $\begin{array}{c}\text { TGO } \\
\text { (UKat/Lt) }\end{array}$ & $\begin{array}{c}\text { TGP } \\
\text { (UKat/Lt) }\end{array}$ & $\begin{array}{l}\text { Albúmina } \\
\text { sérica }(g r / L t)\end{array}$ \\
\hline FV & 20 & 18800 & 22.0 & 2830 & 16.20 & 4.70 & 0.90 & - \\
\hline VM & 27 & 3500 & 11.0 & 26 & 8.43 & 0.98 & 0.46 & 38.0 \\
\hline $\mathrm{HC}$ & 29 & 29300 & 19.7 & - & 1.22 & 0.65 & 0.16 & 31.3 \\
\hline $\mathrm{HM}$ & 29 & 7600 & 22.0 & 70 & 1.11 & 1.98 & 0.31 & 33.0 \\
\hline TR & 24 & 8200 & 14.3 & 36 & 6.10 & 3.43 & 1.10 & 40.0 \\
\hline PR & 23 & 16200 & 11.0 & 20 & 7.66 & 3.75 & 0.66 & 38.0 \\
\hline $\mathrm{MCH}$ & 25 & 6100 & 42.7 & 22 & 26.58 & 0.31 & 0.13 & - \\
\hline $\mathrm{CCQ}$ & 34 & 9600 & 14.2 & 93 & 4.21 & 2.13 & 0.75 & 42.0 \\
\hline $\mathrm{FC}$ & 25 & 13000 & - & - & 8.88 & 0.76 & 0.66 & - \\
\hline LE & 36 & 10200 & 17.0 & 27 & 3.44 & 7.15 & 1.35 & 33.0 \\
\hline VM & 27 & 17100 & 16.1 & 37 & 6.66 & 3.13 & 0.50 & 39.0 \\
\hline BJ & 29 & 19700 & 13.1 & 23 & 12.93 & 1.06 & 0.43 & - \\
\hline $\mathrm{CHC}$ & 30 & 12500 & - & - & 3.60 & 1.28 & 0.28 & - \\
\hline
\end{tabular}

Hcto: Hematocrito. TP: Tiempo de Protrombina. TTP: Tiempo parcial de Tromboplastina.

TGO: Transaminasa glutámico-axalacética. TGP: Transaminasa Glutámico-piruvica.

Tabla No4. Electrolitos séricos y urinarios.

\begin{tabular}{|c|c|c|c|c|c|c|c|c|c|c|c|}
\hline PAC & $\begin{array}{c}\text { Urea } \\
(\mathrm{mmol} / \mathrm{Lt})\end{array}$ & $\begin{array}{c}\text { Creatinina } \\
(\mu \mathrm{mol} / \mathrm{Lt})\end{array}$ & $\begin{array}{l}\text { Na sérico } \\
(\mathrm{mmol} / \mathrm{Lt})\end{array}$ & $\begin{array}{l}\text { K sérico } \\
(\mathrm{mmol} / \mathrm{Lt})\end{array}$ & $\begin{array}{l}\mathrm{Cl} \text { sérico } \\
(\mathrm{mmol} / \mathrm{Lt})\end{array}$ & $\begin{array}{l}\text { Na urin } \\
(\mathrm{mmol} / \mathrm{Lt})\end{array}$ & $\begin{array}{c}\text { K urin } \\
(\mathrm{mmol} / \mathrm{Lt})\end{array}$ & $\begin{array}{c}\mathrm{Cl} \text { urin } \\
(\mathrm{mmol} / \mathrm{Lt})\end{array}$ & $\begin{array}{l}\text { Osm sérica } \\
(\mathrm{mosm} / \mathrm{Kg})\end{array}$ & $\begin{array}{l}\text { Osm urin } \\
(\mathrm{mosm} / \mathrm{Kg})\end{array}$ & $\begin{array}{l}\mathrm{Mg} \text { sérico } \\
(\mathrm{mmol} / \mathrm{Lt})\end{array}$ \\
\hline $\mathrm{FV}$ & 10.8 & 150.7 & 117 & 2.2 & 103 & - & - & - & 326 & 369 & 0.7 \\
\hline VM & 3.2 & 132.6 & 127 & 2.4 & 95 & 68 & 17 & 92 & 272 & 573 & 0.7 \\
\hline $\mathrm{HC}$ & 9.9 & 114.9 & 153 & 5.3 & 121 & - & - & - & - & - & - \\
\hline HM & 8.5 & 150.2 & 140 & 3.0 & 106 & - & - & - & - & - & - \\
\hline TR & 4.2 & 73.0 & 133 & 3.1 & 100 & - & - & - & 274 & 217 & - \\
\hline PR & 2.6 & 61.8 & 114 & 3.1 & 82 & 88 & 49 & 117 & 248 & 438 & 0.9 \\
\hline $\mathrm{MCH}$ & 3.4 & 141.4 & 123 & 3.8 & 104 & 43 & 15 & 40 & 304 & 329 & 0.7 \\
\hline $\operatorname{CCQ}$ & 12.2 & 163.6 & 124 & 1.9 & 100 & 41 & 8 & 35 & 288 & 346 & 0.9 \\
\hline FC & 1.9 & 44.2 & 118 & 2.0 & 81 & - & - & - & - & - & 0.9 \\
\hline LE & 2.2 & 53.0 & 126 & 2.8 & 97 & $<10$ & 139 & 24 & 263 & 540 & 0.8 \\
\hline VM & 7.2 & 114.9 & 130 & 2.3 & 110 & 181 & 19 & 145 & 284 & 329 & 0.6 \\
\hline BJ & 6.7 & 61.8 & 126 & 2.5 & 100 & $<10$ & 20 & 53 & 279 & 370 & 0.8 \\
\hline $\mathrm{CHC}$ & 7.5 & 70.7 & 127 & 3.5 & 103 & - & - & - & - & - & 0.8 \\
\hline
\end{tabular}




\begin{tabular}{|c|c|c|c|c|c|}
\hline \multirow[b]{2}{*}{ PACIENTE } & \multicolumn{4}{|c|}{ Tabla N05. Gases sanguíneos. } & \multirow[b]{2}{*}{$E B$} \\
\hline & $\mathrm{pH}$ & $\mathrm{PCO} 2$ & P02 & $\mathrm{HCO} 3$ & \\
\hline $\mathrm{FV}$ & 7.05 & 20 & 108 & 5.5 & -23.9 \\
\hline VM & 7.54 & 14 & 194 & 12.0 & -5.5 \\
\hline $\mathrm{HC}$ & 7.18 & 31 & 82 & 11.3 & -16.0 \\
\hline HM & 7.00 & 20 & 100 & 6.5 & -21.0 \\
\hline TR & 7.29 & 25 & 46 & 12.1 & -12.3 \\
\hline$P R$ & 7.56 & 14 & 288 & 12.0 & -5.0 \\
\hline $\mathrm{MCH}$ & 7.20 & 12 & 188 & 4.8 & -20.3 \\
\hline $\operatorname{CCQ}$ & 7.36 & 15 & 171 & 8.4 & -13.3 \\
\hline FC & 7.55 & 16 & 170 & 13.9 & -3.8 \\
\hline LE & 7.37 & 21 & 74 & 12.1 & -10.2 \\
\hline VM & 7.17 & 14 & 106 & 7.9 & -13.4 \\
\hline BJ & 7.06 & 35 & 79 & 9.6 & -20.4 \\
\hline $\mathrm{CHC}$ & 7.42 & 11 & 117 & 6.8 & -12.3 \\
\hline
\end{tabular}

Tabla Nº. Tratamiento endovenoso inicial y condición final de los pacientes.

\begin{tabular}{lccc} 
PACIENTE & $\begin{array}{c}\text { SOLUCIONES EV } \\
\text { TRATAMIENTO INICIAL }\end{array}$ & $\begin{array}{c}\text { DIAS DE } \\
\text { HOSPITALIZACION }\end{array}$ & $\begin{array}{c}\text { CONDICION } \\
\text { FINAL }\end{array}$ \\
\hline FV & Hipertónica, Hipotónica, Isotónica & 1 & Fallecido \\
MCH & Hipertónica, Hipotónica, Isotónica. & 1 & Fallecido \\
HC & Hipertónica, Hipotónica, Isótonica. & 3 & Fallecido \\
HM & Isotónica, Hipotónica. & 1 & Fallecido \\
VM & Hipertónica, Hipotónica, Isotónica. & 2 & Vivo con secuelas \\
LE & Hipertónica, Hipotónica, Isotónica. & 10 & Vivo con secuelas \\
VM & Hipertónica, Hipotónica, Isotónica. & 32 & Vivo con secuelas \\
PR & Isotónica, Hipotónica. & 17 & Vivo con secuelas \\
CCQ & Isotónica, Hipotónica. & 6 & Vivo sin secuelas \\
BJ & Isotónica, Hipotónica. & 7 & Vivo sin secuelas \\
FC & Hipertónica, Hipotónica. & 17 & Vivo sin secuelas \\
CHC & Isotónica, Hipotónica. & 5 & Vivo sin secuelas \\
TR & Hipotónica & 4 & Vivo sin secuelas \\
\hline
\end{tabular}

Tabla N%. Variables predictoras de la condición final de los pacientes.

\begin{tabular}{llccc}
$\begin{array}{l}\text { Variable } \\
\text { dependiente }\end{array}$ & $\begin{array}{l}\text { Variable } \\
\text { Independiente }\end{array}$ & $\begin{array}{c}\text { Chi } \\
\text { cuadrado }\end{array}$ & Likelihood & $\begin{array}{c}\text { Prueba Exacta } \\
\text { de Fisher }\end{array}$ \\
\hline Condición Final & Tiempo de Protrombina & 0.026 & 0.013 & 0.061 \\
Condición Final & Tipo de Solución & 0.002 & 0.001 & 0.007 \\
& usada a la admisión & & & \\
Condición Final & Relación & 0.053 & 0.047 & 0.086 \\
& urea/creatinina $<40$ & & &
\end{tabular}

$p<0.005$ convencional, aunque otras etiologías bacterianas inusuales o virales no la podemos descartar.

Los pacientes que aquí presentamos se ajustan estrictamente a la definición de golpe de calor, es decir temperatura rectal superior a los $39^{\circ} \mathrm{C}$ persistente por más de 6 horas y que no pudo controlarse por medios convencionales farmacológicos o físicos. Estos pacientes no estaban adaptados a situaciones de alta temperatura ambiental. Los pacientes fueron, en su gran mayoría niños previamente sanos y con un tiempo de enfermedad relativamente corto (menor o igual a 72 horas en el 75\%). En el aspecto clínico, los signos más frecuentes registrados fueron el compromiso del sensorio asociado a la presencia de convulsiones, deshidratación, EDA, dificultad respiratoria y poliuria.

La literatura define este cuadro clínico en forma incompleta, no mencionándose a la poliuria y a la dificultad respiratoria como manifestaciones importantes.

Entre los datos de laboratorio más relevantes, cabe mencionar anemia, leucocitosis, TP prolongado, hiperglicemia y elevación de la transaminasa glutámico oxalacética. Estos hallazgos han sido descritos como parte del Síndrome de Golpe de calor en una serie de casos de pacientes adultos expuestos a ejercicios intensos $(9,10,11,12)$.

Como alteraciones del medio interno hemos observado hiponatremia en el $84 \%$ de los pacientes, hipocalcemia en el $70 \%$, creatinina elevada para la edad en el $84 \%$, retención nitrogenada en el 53\%, acidosis metabólica en el 100\% (con Anion Gap elevado en el $70 \%$ ). Estos resultados son acordes con lo observado en pacientes adultos $(13,14)$. En niños pequeños sólo se describen algunos casos como reportes aislados $(15,16,17,18)$. A diferencia de estos estudios, nuestro trabajo muestra una frecuencia más elevada y constante de las anormalidades en la mayoría de los pacientes. La hiponatremia estaría relacionado a secreción inapropiada de la hormona antidiurética producida por el compromiso encefálico ocasionado por la hipertermia. La observación se sustenta en que la gran mayoría de pacientes presentaron osmolalidad urinaria alta y sodio urinario elevado con un aclaramiento de agua libre negativo a pesar de mantener flujos urinarios normales o altos (19).

El análisis estadístico mostró que, el tiempo de protrombina aumentado fue la variable mas importante asociada a mal pronóstico. Es interesante esta información, por cuanto es inédita en la literatura y permitiría al pediatra percatarse de la gravedad del paciente e inferir un riesgo elevado de muerte. 
Las alteraciones del TP podrían ser producto de un daño directo por hipertermia en la función hepática y del endotelio vascular. Actualmente se ha descrito evidencias de disfunción endotelial por injuria celular directa por alteraciones en el tono y permeabilidad vascular (20) y se ha demostrado activación de la "molécula -1" de adhesión intercelular, endotelinas y factor de Von Willebrand.

El uso de múltiples soluciones, sobre todo las que incluyen soluciones hipertónicas, se asoció con mal pronóstico (secuelas y muerte). Creemos que esto ocurre por la velocidad de infusión de la carga osmolal en un paciente lactante con un sistema regulador de la circulación cerebral inmaduro. Esto generaría un alto gradiente osmolal entre el intracelular del sistema nervioso central y el agua plásmatica que ha recibido las soluciones hipertónicas. Este gradiente es muy importante en el lactante, dado que en ellos los mecanismos de generación de osmoles idiogénicos son de lenta producción.

La relación de urea/creatinina elevada en algunos casos parece ser multifactorial, siendo su componente fundamental la redistribución del volumen sanguíneo renal y el estado hipercatabólico, como ya ha sido descrito $(21,22)$.

La poliuria puede estar asociada a la presencia de hipocalemia, la cual ha sido también descrita años atrás (23).

Una visión amplia de la enfermedad permite clasificarla como falla orgánica múltiple (FOM), causada por la excesiva acumulación del calor dentro del cuerpo del niño y su incapacidad para poder disiparla, produciendo daño en diferentes órganos y como corolario final, falla cardiaca $(24,25)$.

Los lactantes son más susceptibles al calor producido ambientalmente por la desproporción entre el área expuesta y la temperatura existente (26), por su mayor metabolismo y por su menor capacidad de sudoración. Además, se debe tener en cuenta que estos niños no estuvieron aclimatados a elevadas temperaturas.

Es nuestra impresión, que un buen enfoque de pacientes lactantes con las características de Golpe de Calor, debe iniciarse con un diagnóstico correcto y una exacta valoración de los factores asociados a riesgo de muerte o daño cerebral y posteriormente, a una intervención terapéutica adecuada, que esté basada en la prevención de la ocurrencia del Golpe de Calor y establezca un plan de tratamiento preconcebido que ayude a salvar la vida del paciente con la menor secuela posible.
De acuerdo a nuestras observaciones, podemos sugerir que en el tratamiento se debe considerar primero corregir la hipertermia para disminuir sus efectos directos. El soporte hidroelectrolítico debería realizarse con soluciones isotónicas asegurando un buen aporte de potasio por la poliuria. Las soluciones hipertónicas se asociaron a mal pronóstico, por lo que la corrección de la hiponatremia debe hacerse lentamente y con un balance hídrico estricto en una sala de cuidados intensivos especializada $(27,28,29)$.

\section{Correspondencia:}

\section{Dr. Reyner Loza Munarriz.}

Servicio de Nefrología, Departamento de Medicina. Hospital Nacional Cayetano Heredia. Av Honorio Delgado s/n. San Martín de Porras. Lima

\section{REFERENCIAS BIBLIOGRAFICAS}

1. Wakefield EG, Hall WW. Heat injuries: A preparatory study for experimental heat stroke. JAMA 1929; 89:9295.

2. Adolph ES, Fulton WB. The effects of exposure to high Temperatures upon the circulation in man. Am J Physiol 1923; 67:573-579.

3. Weiner JS, Horne GO. A classificacion of Heat illness. Br Med J 1958; 1:1533-1535.

4. Dixit SN, Bushary KO, Brooks BR. Epidemic Heat Stroke in a Midwest community risk factors ,neurological complication and secuelae. Wis Med J 1997; 96:39-41.

5. Ishihara K, Szerlip MH. Anion Gap Acidosis in Electrolyte Disorders. Sem Nephrol 1998; 1:83-97.

6. Burton David Rose Introduction to simple and Mixed Acid-base Disorders. In clinical Physiology of AcidBasic and electrolyte Disorders 4th ed Massachusetts: MCGraw-Hill Inc; 1994. pp.500-603.

7. Velázquez JL. Equilibrio Acido Base. en: Alteraciones Hidroelectrolíticas en Pediatría 1era ed. Mexico: Ediciones Medicas del Hospital infantil de México. 1991. pp. 13-21.

8. Instituto Nacional de Salud. Enfermedades inducidas por calor. Lima: Boletín Edición Especial Fenómeno de EL Niño.1998 Enero-Febrero pp. 11-18.

9. Tham MK, Cheng J, Fock KM. Heat Stroke: A Clinical review of 27 cases. Singapore Med J 1989; 30:137-140.

10.Tucker LE, Stanford J,Graves B, Swetnam J, Hamburger S, Anwar a.Classical Heat stroke.Clinical and laboratory assesment. South Med J 1985; 78:20-25.

11.Jimenez-Mejias ME, Montano Díaz M, Villalonga J, Bollain Tienda E. Classical Heat stroke in Spain. Analysis of a series 78 cases. Med Clin(Barc) 1990; 94:481486.

12.Hart GR, Anderson RJ, Crumpler CP, Shulkin A. Epidemical Classical Heat Stroke. Clinical Characteristics and course of 28 patients. Medicine (Baltimore). 1982; 61:189-197. 